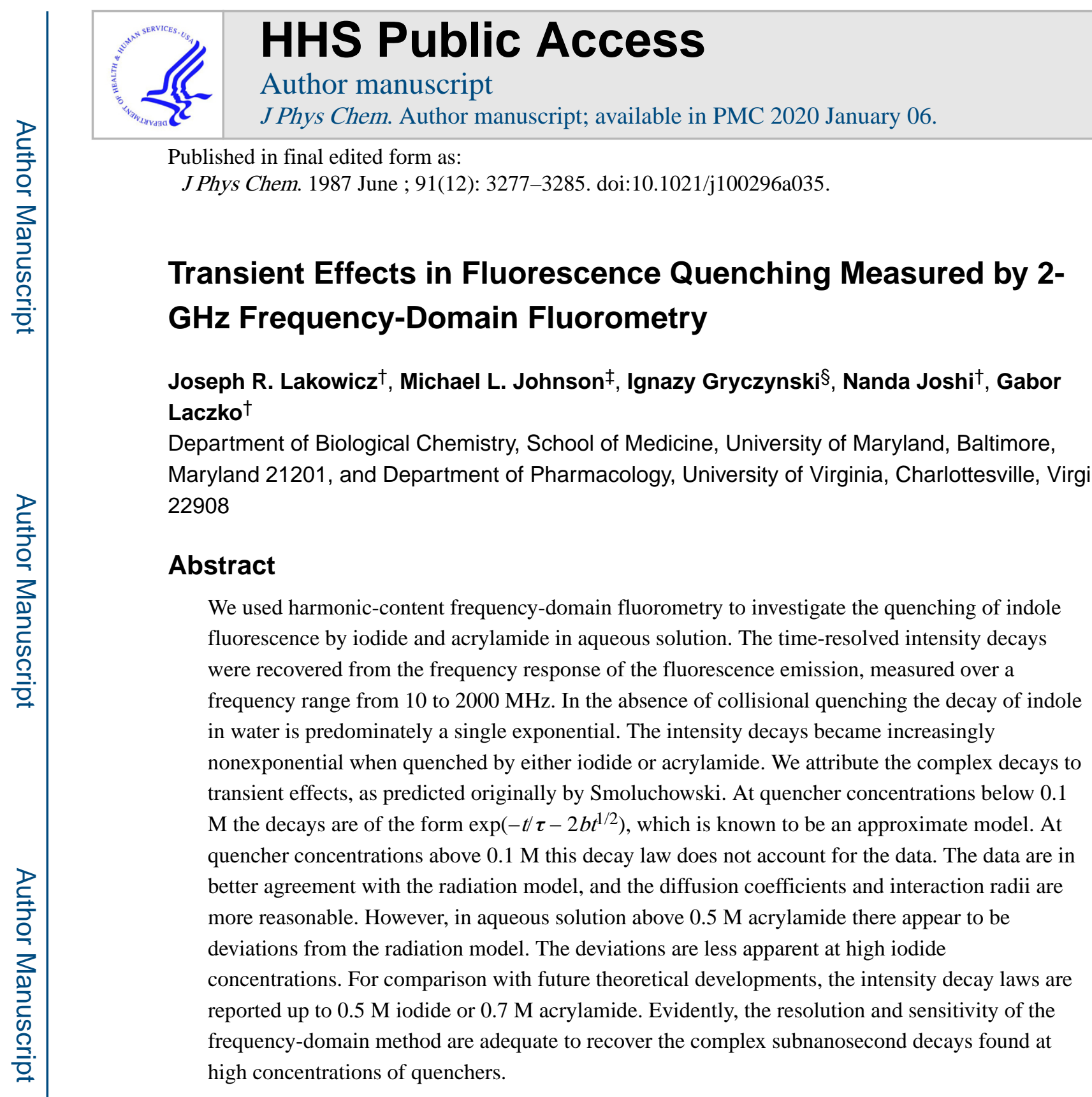

\title{
Registry No.
}

KI, 7681-11-0; indole, 120-72-9; acyrlamide, 79-06-1

\section{Introduction}

Collisional quenching of fluorescence is of considerable interest for physical chemistry and biochemistry. Collisional quenching requires contact between the fluorophore and quencher during the lifetime of the excited state. Consequently, the quenching process yields information about diffusive motions in solution and the accessibility of fluorophores in

\footnotetext{
§Permanent address: Institute of Experimental Physics, University of Gdansk, Gdansk, Poland.

†University of Maryland.

屯University of Virginia.
} 
macromolecules to externally added quenchers. For example, quenching has been used to determine the fraction of protein fluorescence (tryptophan) which is accessible to watersoluble quenchers ${ }^{1-3}$ and the effects of local charge on the approach of quenchers to the fluorophores. ${ }^{1,4}$ Fluorescence quenching has also contributed to our understanding of the dynamics of proteins and membranes, as revealed by the ability of oxygen, iodide, acrylamide, and other quenchers to diffuse through the macromolecules. ${ }^{5-9}$

Collisional quenching is also of interest in physical chemistry. Much of this interest originates with the well-known positive deviations from the Stern-Volmer equation. ${ }^{10,11}$ The frequency of the quenching events is expected to depend upon the diffusion coefficients of the probe and quencher, their radius of interaction, electrostatic attraction or repulsion, and the symmetry of their reactive surfaces. To date, most theories and data are for the steadystate behavior of the system, that is, the intensities, yields, or rate constants observed with constant illumination. However, the time-dependent decays of fluorescence can yield additional information on the transient concentration gradients which are created around the ensemble of fluorophores. The excited fluorophore $(\mathrm{F})$ is created instantaneously upon absorption of a photon. Initially, the quenchers $(\mathrm{Q})$ are distributed randomly around the fluorophores. Subsequently, the closer F-Q pairs are extinguished by the collisional encounters, while the fluorophores without a nearby quencher may exist for longer periods of time. Hence, following $\delta$-function excitation the sample evolves so that only the more distant F-Q pairs remain. This evolution is explained by a time-dependent rate constant for quenching. At short times the rate constant is larger, reflecting the interaction of the more closely spaced F-Q pairs. At later times the rate constant decays to the diffusion-controlled limit. To a first approximation the transient effects are expected to modify the decay law from a single exponential $\left(\exp \left(-t / \tau_{0}\right)\right)$, to the form $\left(\exp \left(-t / \tau-2 b t^{1 / 2}\right)\right.$, where $\tau_{0}$ is the decay time in the absence of quenching and $\tau$ is the decay time for the quenched emission if transient effects are not present. ${ }^{12-14}$ If the resolution is adequate, the time-resolved measurements can be shown to contain transient terms with a $t^{1 / 2}$ dependence. Under favorable circumstances, the encounter radius for the F-Q pair and the sum of the diffusion coefficients can be obtained from the data.

In this report we examine quenching of indole by iodide and acrylamide in aqueous solution. Indole was selected because its decay in the absence of quenching is predominately a single exponential, and indole is the dominant fluorophore in proteins. Iodide and acrylamide have been used extensively in studies of protein fluorescence. ${ }^{3}$ Instead of the more familiar timeresolved measurements we use the new technique of frequency-domain fluorometry, ${ }^{15,16}$ including the recent extension to $2 \mathrm{GHz}$ via the harmonic content of a train of picosecond pulses. ${ }^{17,18}$ This technique is known to yield good resolution of complex multiexponential decays ${ }^{19}$ and of more complex time-dependent spectral shifts. ${ }^{20,21}$ Hence, it is of interest to apply this technique to the more complex problem of transient effects in quenching. The frequency responses were measured on samples with up to $0.7 \mathrm{M}$ quencher. The availability of the experimental decays at these high quencher concentrations may be used for comparison with future theory or simulations. 


\section{Theory}

\section{Transient Effects in Quenching.}

Consider a fluorophore which displays a single-exponential decay. Following $\delta$-function excitation the time-dependent intensity is

$$
I(t)=I_{0} \exp \left(-t / \tau_{0}\right)
$$

where $\tau_{0}$ is the lifetime or decay time in the absence of quenching. Suppose the fluorophore is in homogeneous solution and surrounded by randomly distributed quenchers. Predictions of the intensity decays for such samples have been the subject of several significant publications. ${ }^{22-24}$ Our expressions are predominately in the form suggested by Ware and coworkers. ${ }^{12-14}$ Quenching increases the decay rate of the excited state by a rate constant $k(t)$, which is itself time-dependent. The form of $k(t)$ is complex, and depends upon the assumptions used in its derivation. The simplest case is obtained by assuming the concentration gradient around the fluorophore is a step function which increases from 0 to the bulk concentration $[\mathrm{Q}]$ at the interaction radius $R$. This radius is taken as the sum of the radii of the fluorophore and the quencher. This assumption means the fluorophore is quenched instantaneously when the quencher diffuses to the boundary at $r=R$. Then, the time-dependent rate constant is given by

$$
k(t)=4 \pi D N^{\prime} R\left(1+\frac{R}{(\pi D t)^{1 / 2}}\right)
$$

where $N^{\prime}$ is $6.02 \times 10^{20}$ and $D$ is the sum of the diffusion coefficients. Evidently, the transient effects will be largest for small values of $t$ and for slow diffusion. For instance, for $R=8 \AA$ and $D=1 \times 10^{-5} \mathrm{~cm}^{2} / \mathrm{s}$ the value of $k(t)$ is 1.23 -fold larger at $t=4 \mathrm{~ns}$ than in the absence of transient effects. If the diffusion coefficient is reduced 10 -fold to $1 \times 10^{-6} \mathrm{~cm}^{2} / \mathrm{s}$, then at $4 \mathrm{~ns} k(t)=1.7$-fold larger than in the absence of transient effects. If $D=0.2 \times 10^{-6}$ then $k(t)$ is 2.7 -fold larger. In our aqueous samples the diffusion coefficients are near $10^{-5}$ $\mathrm{cm}^{2} / \mathrm{s}$, which results in smaller transient effects than we could have observed in more viscous solutions.

The intensity decay is found by solving

$$
\frac{\mathrm{d} I(t)}{\mathrm{d} t}=-\left[\frac{t}{\tau_{0}}+k(t)[\mathrm{Q}]\right] I(t)
$$

The general solution for any $k(t)$ is

$$
I(t)=I_{0} \exp \left(-\frac{t}{\tau_{0}}\right) \exp \left[-[\mathrm{Q}] \int_{t=0}^{t} k(t) \mathrm{d} t\right]
$$

For the time-dependent quenching constant given in eq 2 the solution is 


$$
I(t)=I_{0} \exp \left[-\frac{t}{\tau_{0}}-4 \pi D R N^{\prime}[\mathrm{Q}] t\left(1+\frac{2 R}{(\pi D t)^{1 / 2}}\right)\right]
$$

This equation can be written as

$$
I(t)=I_{0} \exp \left(-t / \tau-2 b t^{1 / 2}\right)
$$

where $\tau$ is the decay time expected in the absence of transient effects

$$
\frac{1}{\tau}=\frac{1}{\tau_{0}}+4 \pi R D N^{\prime}[\mathrm{Q}]
$$

and

$$
b=4 R^{2} N^{\prime}(\pi D)^{1 / 2}[\mathrm{Q}]
$$

Hence the intensity decay is expected to become more complex than a single exponential, and the additional term is dependent on $t^{1 / 2}$. The values of $R$ obtained from the quenching data are often larger than the values calculated from the molecular structures. Hence, eq 5 is sometimes modified by replacing $R$ with $R^{\prime}$, where $R^{\prime}$ is an apparent encounter radius. ${ }^{13,25}$ Furthermore, eq 5 has been used as an approximation to the more complete theory based on the radiation boundary conditions (below).

Examination of eq 2 and 5 reveals that for long times the transient terms approach zero. Then, the quenching constant becomes diffusion controlled and independent of time

$$
k=k(t)=4 \pi N^{\prime} D R
$$

and the intensity decay is again a single exponential

$$
I(t)=I_{0} \exp \left(-\frac{t}{\tau_{0}}-k[\mathrm{Q}] t\right)
$$

Equations 9 and 10 are the usual expressions for analysis of quenching data without consideration of transient effects and lead to the Stern-Volmer equation. For purely collisional quenching the decay time is $\tau^{-1}=\tau_{0}^{-1}+k[\mathrm{Q}]$, which on rearrangement yields

$$
\frac{\tau_{0}}{\tau}=1+k \tau_{0}[\mathrm{Q}]=\frac{F_{\mathrm{o}}}{F}
$$

For collisional quenching the intensities $\left(F_{\mathrm{o}}\right.$ and $\left.F\right)$ are proportional to the decay times, so $F \mathrm{o} / F$ is expected to be equivalent to $\tau_{0} / \tau$.

Collins and Kimball ${ }^{22}$ pointed out several difficulties with eq 2 , the most obvious being that the quenching constant is infinite at $t=0$. To avoid this difficulty the infinite concentration gradient at $r=R$ is softened by assuming that the rate of quenching is a finite value at the 
distance $R$. These "radiation" boundary conditions yield a more complex form for $k(t)$. $13,22,23$

$$
\begin{gathered}
k(t)=\frac{4 \pi R D N^{\prime}}{1+D / \kappa R}\left[1+\frac{\kappa R}{D} \exp \left(x^{2}\right) \operatorname{erfc}(x)\right] \\
x=\frac{(D t)^{1 / 2}}{R}\left[1+\frac{\kappa R}{D}\right]
\end{gathered}
$$

This expression introduces a new parameter $\mathrm{k}$, which is a specific rate constant for quenching with units of centimeters per second. The essential point is that eq 2 and 5 are thought to be only approximately correct, whereas eq 12 is thought to account for obvious flaws in the theory. At quencher concentrations above $0.1 \mathrm{M}$ our data show substantial deviations from eq 2 and 5, and the data are better fit by eq 12 and 13. Additionally, we suspect our data for acrylamide concentrations above $0.5 \mathrm{M}$ reveal inadequacies in eq 12 and 13 and hence contain information on the more subtle aspects of quenching.

\section{Frequency-Domain Fluorometry.}

The intensity decays were examined in the frequency domain. The samples are excited with amplitude-modulated light, over a range of modulation frequencies from 10 to $2000 \mathrm{MHz}$. At each frequency $(\omega)$, we measure the phase delay $\left(\phi_{\omega}\right)$ and the extent of demodulation $\left(m_{\omega}\right)$ of the emission, each relative to the incident light. The measured values are compared with those predicted from an assumed decay law, and the parameters chosen are those which minimize the squared deviations. We analyzed the frequency responses of the samples using a sum of exponentials, the expression with a $t^{1 / 2}$ dependence (eq 6), and the more complex decay predicted by eq 4,12 , and 13 . For a sum of exponentials the intensity decay is described by

$$
I(t)=\sum_{i} \alpha_{i} e^{-t / \tau} i
$$

The fractional contribution of each decay time to the steady-state intensity is

$$
f_{i}=\frac{\alpha_{i} \tau_{i}}{\sum_{i} \alpha_{i} \tau_{i}}
$$

We used the "sum of exponentials" analysis to provide a parametrized form of intensity decay for future comparisons with theory. For any form of the decay law the phase and modulation values are given by

$$
\begin{gathered}
\phi_{\omega}=\arctan \left(N_{\omega} / D_{\omega}\right) \\
m_{\omega}=\left(N_{\omega}^{2}+D_{\omega}^{2}\right)^{1 / 2}
\end{gathered}
$$

where 


$$
\begin{aligned}
& N_{\omega}=\int_{0}^{\infty} I(t) \sin \omega t \mathrm{~d} t / \int_{0}^{\infty} I(t) \mathrm{d} t \\
& D_{\omega}=\int_{0}^{\infty} I(t) \cos \omega t \mathrm{~d} t / \int_{0}^{\infty} I(t) \mathrm{d} t
\end{aligned}
$$

For multiexponential decays (eq 14) we use analytical expressions for the transforms. For the other decay laws we were unable to find analytical expressions for the sine and cosine transforms. Hence, numerical integration was used. In particular we used an adaptive Newton-Cotes 9-point integration. ${ }^{26}$ It was important to match the time range of the integral to that of the intensity decay, especially at high concentrations of quenchers where the transient effects are of larger amplitude. For the radiation boundary conditions (eq 12 and 13)) the term $f(x)=\exp \left(x^{2}\right) \operatorname{erfc}(x)$ was obtained during the iterations from a look-up table. The tabulated values were obtained from the International Mathematics and Statistics Library. ${ }^{27}$ Values of $f(x)$ for $x$ values between those in the look-up table were obtained by using spline interpolation. ${ }^{26}$

The goodness of fit is characterized by

$$
\chi_{R}{ }^{2}=\frac{1}{\nu} \sum_{\omega}\left[\frac{\left(\phi_{\omega}-\phi_{\mathrm{c} \omega}\right)}{\delta \phi}\right]^{2}+\frac{1}{\nu} \sum_{\omega}\left[\frac{\left(m_{\omega}-m_{\mathrm{c} \omega}\right)}{\delta m}\right]^{2}
$$

where $v$ is the number of degrees of freedom, and $\delta \phi$ and $\delta m$ are the experimental uncertainties in the phase and modulation values, respectively. If $\delta \phi$ and $\delta m$ are accurately known, then $\chi_{R}{ }^{2}$ is expected to be near unity for a correct or adequate intensity decay model. In practice, the values of $\delta \phi$ and $\delta \mathrm{m}$ are not precisely known, and these vary about 2 fold depending on the experimental details. However, the relative values of $\chi_{\mathrm{R}}{ }^{2}$ can always be compared for various models.

An important aspect of the analysis is estimation of the uncertainties in the recovered parameters. The uncertainties are usually estimated from the diagonal terms of the covariance matrix. ${ }^{28}$ However, it is known that these uncertainties are underestimates because the procedure assumes uncorrelated parameters. Our algorithm accounts for parameter correlation in estimation of the uncertainties ${ }^{29,30}$ and will be the subject of a separate publication. ${ }^{31}$

\section{Materials and Methods}

Frequency-domain data were obtained on the instrument described previously. ${ }^{16,17}$ The excitation source was the 3.7931-MHz cavity-dumped output from a Coherent Model 700 dye laser. The dye was rhodamine 6G. The second jet was used with the saturable absorber DODCI (3,3' -diethyl-2,2' -oxadicarbocyanine iodide). The dye laser was synchronously pumped at $75.86 \mathrm{MHz}$ using a mode-locked argon ion laser, Coherent Innova 15, about 800 $\mathrm{mW}$ at $514 \mathrm{~nm}$. The visible cavity-dumped output of the dye laser was typically near 60 $\mathrm{mW}$. To obtain $295 \mathrm{~nm}$ for excitation of indole we used a Spectra Physics Model 390 
frequency doubler, with a KDP angle-tuned crystal. The average UV power was near 0.5 $\mathrm{mW}$, which was attenuated 50- to 100-fold prior to the sample. The pulse width of the visible output of the dye laser was $5 \mathrm{ps}$ or less. This source is intrinsically modulated to about $50 \mathrm{GHz}$.

Detection of the forced response at each frequency is accomplished with a microchannel plate PMT (Hammamatsu R1564), which provides an upper frequency limit of $2 \mathrm{GHz} .{ }^{17}$ The PMT is externally cross-correlated to reject extraneous signals, to improve the signal-tonoise ratio, and to provide a low-frequency signal which contains the phase and modulation information. The excitation wavelength was $295 \mathrm{~nm}$, and the emission was observed through a Schott WG320 filter to eliminate scattered light. The excitation was polarized vertically, and the emission was viewed through a polarizer at $54.7^{\circ}$ from the vertical. These conditions eliminate the effects of rotational diffusion on the intensity decay. ${ }^{32}$ For all measurements we used scattered light as the reference and not a reference fluorophore. ${ }^{54} \mathrm{~A}$ reference fluorophore was not needed because the time response of the microchannel plate PMT does not depend on wavelength. ${ }^{55}$ Also, extensive testing of this instrument did not reveal any effects of wavelength on the frequency response [ref 17, Figure 9].

Unless stated otherwise all samples were in aqueous buffer, $0.05 \mathrm{M}$ Tris, $\mathrm{pH} 7.5,20{ }^{\circ} \mathrm{C}$. For iodide quenching the samples contained $\mathrm{KCl}$ and/or $\mathrm{KI}$, for a total concentration of $0.50 \mathrm{M}$. These samples also contained $10^{-3} \mathrm{Na}_{2} \mathrm{~S}_{2} \mathrm{O}_{3}$ to prevent formation of iodine. Acrylamide was from Aldrich, Gold Label, lot HMO2820KL. For acrylamide quenching no other salts were added. The emission spectra of all indole samples were examined and found to be characteristic of indole, irrespective of the concentration of iodide or acrylamide. Quenching resulted in decreased intensity, but did not result in any spectral shift. Blank samples were examined in the frequency-domain instrument. The background fluorescence and/or scatter was typically $0.5 \%$ and in no case did it exceed $1 \%$.

\section{Results}

\section{Intensity Decay of Indole in the Absence of Quenching.}

It is instructive to examine the frequency-domain data for indole in the absence of quenching. The frequency responses are shown at temperatures from 5 to $50^{\circ} \mathrm{C}$ (Figure 1). As the modulation frequency is increased the phase angles increase and the modulation decreases. The lifetime of indole decreases with increasing temperature, which results in displacement of the curves to higher frequency. The parameters describing the intensity decay are obtained by the method of nonlinear least squares. A decay law is assumed, and the parameters are varied to obtained the best fit of the theoretical curve (solid lines) to the data (symbols). For indole from 5 to $50^{\circ} \mathrm{C}$ the intensity decay is adequately described by a single-exponential decay law. The single-exponential curves closely match the data.

Differences between the data and the curves are more readily seen by examination of the deviations between the measured and calculated values. The deviations are mostly small $\left(<0.5^{\circ}\right.$ and $<1 \%$ in modulation), which indicates the single-exponential model is adequate to explain the data. 
The parameters describing the decays are summarized in Table I. For the single-exponential fits the values of $\chi_{\mathrm{R}}{ }^{2}$ range from 1.3 to 2.6 . While the latter value may be considered too large we stress that the precise values of the uncertainties $(\delta \phi$ and $\delta m)$ are not known. Our criterion for selecting a decay law is based on comparison of various models for a single set of data. The indole data were analyzed by using the double-exponential model (eq 14 with $I$ $=2$ ). The values of $\chi_{R}{ }^{2}$ did not decrease significantly, indicating the second component is not required by the data. Additionally, the amplitude of the second component is small. It is particularly informative to examine the uncertainties of the parameters, including the effects of parameter correlation. ${ }^{29,30}$ It should be noted that the range of decay times consistent with the data is quite large for the smaller amplitude component, often overlapping with the value of the dominant decay time. Based on these observations and previous experience, we conclude the decay of indole is adequately described as a single exponential. A singleexponential decay is intuitively useful because the transient effects in quenching are expected to induce nonexponential decays. While our fitting algorithm can analyze for transient effects with multiexponential decays of the unquenched fluorophore, it is more difficult to see the transient effects in the data.

\section{Stern-Volmer Plots for Quenching.}

Quenching data are most frequently presented as Stern-Volmer plots, which are shown for iodide (Figure 2) and acrylamide quenching of indole (Figure 3). Also shown are the emission spectra, which are decreased in amplitude but not altered in shape by quenching. The slopes of these plots are the Stern-Volmer quenching constants, which were found to be 31 and $33 \mathrm{M}^{-1}$ for iodide and acrylamide, respectively. These values are in good agreement with previously published values. 3,4

Also shown are the values of $\tau_{0} / \tau$, where $\tau$ is the best single-exponential fit to the frequency response. In this case the Stern-Volmer plots show substantial positive deviations. These deviations are probably due to the increasing proportion of the decay occurring as a shortlived component (below). A more linear Stern-Volmer plot was found when the mean decay times were used $\left.(\langle\tau\rangle)=f_{1} \tau_{1},+f_{2} \tau_{2}\right)$, and the $\tau_{0} / \tau$ ratios were then in good agreement with the intensity ratios. The positive deviations observed with the single decay time values illustrate the difficulties in using intensity decay data which are not adequate to recover the detailed form of the decay. The single decay time values represent an average over the true decay, which is weighted in a complex and unknown manner by the time resolution of the measurements. It seems uninformative to attempt to explain such positive deviations based on apparent single decay times.

\section{Detection of Nonexponential Decays in the Presence of Quenching.}

The frequency responses of the indole samples were measured over a range of quencher concentrations from 0 to $0.7 \mathrm{M}$. Typical data are shown in Figures 4 and 5 for indole in the presence of iodide and acrylamide, respectively. Also shown are the best single-exponential fits to the data. In the presence of quencher the measured frequency response cannot be accounted for by a single-exponential decay, as is seen from the large and systematic deviations between the data and the best single-exponential fit ( $\bullet$, lower panels). In the absence of quenching the deviations are small and randomly distributed (O). At almost any 
concentration of quencher over $0.025 \mathrm{M}$, which results in quenching by about 2-fold, the decays becomes more complex than a single exponential. For instance, even for a 2 -fold decrease in the decay time, $\chi_{\mathrm{R}}{ }^{2}$ increases 3 - to 8 -fold (Tables II and III). With our approximate 50 degrees of freedom a 2-fold increase in $\chi_{\mathrm{R}}{ }^{2}$ is adequate to reject the model yielding the higher $\chi_{\mathrm{R}}{ }^{2}$ with a certainty of $99 \% .28$ For the higher quencher concentrations the deviations from the single-exponential fit are still greater (Figures 4 and 5), yielding $\chi_{\mathrm{R}}{ }^{2}$ values ranging to several hundred. Examination of the single-exponential fits in Tables II and III shows that the values of $\chi_{\mathrm{R}}{ }^{2}$ increase progressively and dramatically with increases in the quencher concentration. There is little doubt from these results that the quenching process results in more complex decays.

It is of interest to analyze these data by using the multiexponential model (eq 15). The results of this analysis are summarized in Tables II and III. The multiexponential model provides an adequate fit to the data at all quencher concentrations. As the quencher concentration increases both apparent decay times are decreased. Also, the fractional contribution of the shorter decay time becomes more pronounced. In general, the three decay analysis resulted in only a minor improvement in $\chi_{\mathrm{R}}{ }^{2}$ (not shown), and two of the three decay times were usually of similar magnitude. Evidently, the double-exponential model is powerful enough to account for transient effects in intensity decay. It is probable that the intensity decays are more complex than the double exponential at the higher quencher concentrations, but these more complex decays were not unambiguously required to fit our data. Of course, it is difficult to resolve the closely spaced decay times for decay times near $100 \mathrm{ps}$. It is probable the double-exponential model is successful because quenching accelerates the overall decay. If a component remained with the original unquenched decay time, then we believe this would have resulted in the need for a triple-exponential analysis. Additionally, it was necessary to use three decay times to account for the frequency response of indole quenched by acrylamide in the more viscous solvent propylene glycol (not shown).

The decay parameters are informative because they reveal the increased complexity of the decay with increasing amounts of quenching. It is not presently possible to relate these parameters to the molecular parameters which define diffusive transport in these systems. However, these intensity decay parameters may be compared numerically with theories or simulations of collisional quenching.

\section{Smoluchowski Analysis of Indole Quenching.}

The frequency-domain data were analyzed by using the decay law expected from the Smoluchowski theory for quenching (eq 6 and 7). It should be noted that this model is more restrictive than the double-exponential model in that it contains only one additional parameter $(b)$ over a single-exponential decay, whereas the double-exponential model has two additional parameters $\left(\tau_{2}\right.$ and $\left.a_{2}\right)$. The Smoluchowski approximation was found to provide good fits to the data when the degree of quenching was less than 4-fold. This is seen from the rather good fits obtained with eq 7 and the modest values of $\chi_{\mathrm{R}}{ }^{2}$ (Tables IV and V), which are comparable to that obtained by using the double-exponential model. These results indicate that eq 7 accounts for decay of quenched indole, at least for 4-fold quenching or less. 
At low quencher concentration the parameters recovered from the analysis are reasonable in consideration of the size and molecular weights of the diffusing species. The apparent interaction radii for indole and iodide or acrylamide are near 7 and $9 \AA$, respectively. The indole-acrylamide radius is in close agreement with the value of 6-7 $\AA$ calculated from the structures. ${ }^{33}$ The apparent diffusion coefficients are near 0.7 and $1.2 \times 10^{-5} \mathrm{~cm}^{2} / \mathrm{s}$. The apparent diffusion coefficient of acrylamide is close to that of similarly sized molecules such as propanol or acetamide in water, $1 \times 10^{-5} \mathrm{~cm}^{2} / \mathrm{s}^{34,35}$ However, the apparent diffusion coefficient of iodide is less than one-half of the KI diffusion coefficient, $1.8 \times 10^{-5} \mathrm{~cm}^{2} / \mathrm{s}$ at $25^{\circ}$ C. $^{36}$

The Smoluchowski model $\left(t^{1 / 2}\right)$ becomes inadequate at the higher quencher concentrations (above $150 \mathrm{mM}$ ). This is evident from the values of $R, D$, and $\chi_{\mathrm{R}}{ }^{2}$ in Tables IV and V. As the quencher concentration increases, the apparent value of $R$ increases, the apparent value of $D$ decreases, and $\chi_{\mathrm{R}}{ }^{2}$ increases. At the highest iodide or acrylamide concentrations the value of $\chi_{\mathrm{R}}{ }^{2}$ increases 33- or 50-fold, respectively, over the values at the lower quencher concentrations. These increases are highly significant with the approximate 50 degrees of freedom. These systematic changes in $R$ and $D$ are greater than the estimated uncertainties in the parameters (Tables IV and V). The apparent values of $R$ and $D$ change with quencher concentration to an extent greater than the estimated uncertainties.

We questioned whether the sets of data for all quencher concentrations were consistent with the Smoluchowski model. The data for a range of quencher concentrations were analyzed globally with $R$ and $D$ as the floating parameters. If we use only the data to $0.075 \mathrm{M}$ of quencher, then the three or four data sets are explained by eq 6 , as seen by the reasonable values of $\chi_{\mathrm{R}}{ }^{2}$ (Tables IV and V). However, if the data for the higher quencher concentrations are used, then the data are not consistent with the Smoluchowski model. The values of $\chi_{\mathrm{R}}{ }^{2}$ are elevated about 20 -fold, which is obviously unacceptable with the approximate 350 degrees of freedom for these global fits. We conclude that the data are not consistent with the Smoluchowski model. Hence, the resolution available from the frequency-domain data provides details on the higher order transient effects in fluorescence quenching.

\section{Radiation Model Analysis.}

The data were then analyzed by using the radiation model, resulting in a considerably improved match between data and the predicted values. This is seen in Figures 6 and 7, where we compared the fits for both models. For the Smoluchowski model we held the interaction radius constant at $7 \AA$, rather than using the larger and unrealistic values of $R$ which are found for the minimum value of $\chi_{R}^{2}$. The data cannot be fit by $\exp \left(-t / \tau-2 b t^{1 / 2}\right)$, as is seen by the mismatch of the theory (---) to the data, and by the large and systematic deviations between theory and data (lower panels). The radiation model (-) provides a considerable improvement in the fit and smaller deviations $(\bullet)$ for quenching by either iodide or acrylamide.

The improved fit provided by the radiation model is also evident from the values of $R$ and $D$ (Tables IV and V). We found that and $R$ were highly correlated in the analysis, so it was usually not possible to obtain reliable fits if $\kappa, R$, and $D$ were all floating parameters. We 
choose $\kappa=250 \mathrm{~cm} / \mathrm{s}$ for both quenchers by examining the values of $R$ and $D$ from the fit, while $\kappa$ was held constant at values ranging from 50 to $500 \mathrm{~cm} / \mathrm{s}$. As $\kappa$ increases $R$ generally decreases. However reasonable values of $R$ near $7 \AA$ were only found for $\kappa$ near $250 \mathrm{~cm} / \mathrm{s}$. Hence, this value was used for all analysis in Tables IV and V.

The most obvious improvement with the radiation model is the recovery of reasonable values of $R$ and $D$ at all quencher concentrations. For iodide quenching, the results appear to be just as expected. The interaction radius is near $7 \AA$, and the diffusion coefficient is essentially equal to the expected value of $1.8 \times 10^{-5} \mathrm{~cm}^{2} / \mathrm{s}$. The values of $\chi_{R}^{2}$ at the highest quencher concentrations are elevated about 3-fold, which is the same elevation found in the multiexponential analysis (Table II). Hence, the elevation in $\chi_{R}^{2}$ is probably intrinsic to the data sets at high quencher concentrations, perhaps reflecting increased levels of random noise in the data from the more highly quenched solutions. However, we suspect the elevated values of $\chi_{R}^{2}$ for the global fits may be the result of the quencher concentration being treated in an approximate manner in this model (see below).

The radiation model yields somewhat less favorable results for acrylamide quenching of indole. The interaction radius is acceptable, but the diffusion coefficients are initially larger than the expected value near 1 to $1.5 \times 10^{-5} \mathrm{~cm}^{2} / \mathrm{s} .{ }^{2,34,35}$ Additionally, the apparent diffusion coefficient decreases with increasing acrylamide concentration. One possible explanation for the larger-than-expected diffusion coefficient could be a rotational component to quenching. Perhaps only a small region of the indole-acrylamide surface is active in quenching. Then rotational diffusion could bring these surfaces into contact. However, this is clearly speculative, and considerably more data and analysis are needed to clarify the meaning of the apparent diffusion coefficients.

At higher quencher concentrations the radiation model seems to fail for acrylamide quenching. This is seen by an elevation in $\chi_{R}^{2}$ above $0.4 \mathrm{M}$ quencher. Since the emission is weakest at the higher quencher concentrations, one could suggest the data contains a higher degree of random noise. We do not think this is the origin of the elevated values of $\chi_{R}^{2}$, as this effect would also elevate $\chi_{R}^{2}$ for the multiexponential fits (Table III), which was not found to occur. Alternatively, we questioned whether the high quencher concentrations could cause changes in viscosity and hence cause a failure of the global fits. We found that the presence of $2 \mathrm{M}$ acrylamide in water increases its viscosity by no more than $5 \%$, which we consider to be too small to cause the poor fits we observed. At present we believe the data for $0.4 \mathrm{M}$ acrylamide or higher display deviations from the radiation model.

Elevated values of $\chi_{R}^{2}$ were also found for global fits by using the data for the higher acrylamide concentrations (Table V). One source of these elevated values of $\chi_{R}^{2}$ could be small errors in the quencher concentrations. However, we do not think this effect is dominant because $\chi_{R}^{2}$ is elevated to a similar extent for both the single concentration analyses and for the global fits. In recent experiments with other fluorophores and quenchers we continue to observe elevated values of $\chi_{R}^{2}$ for global fits with high quencher concentrations (Lakowicz et al., unpublished observations). These observations also argue against errors in the quencher concentrations. We suspect that the radiation model, as currently formulated, is not completely valid for high concentrations of quencher. In fact, it appears that the quencher 
concentration is treated in an approximate manner (see ref 22, eq 22-29). However, we are not aware of a more precise solution of the radiation model, and we suggest that this solution be derived.

It is of interest to interpret the value of $\kappa$, which is the specific rate constant for quenching. This constant can be expressed in the more familiar units of a bimolecular quenching constant $\left(\mathrm{M}^{-1} \mathrm{~s}^{-1}\right)$ by forming the product $\kappa 4 \pi R^{2} N^{\prime} .{ }^{13} \mathrm{Using}$ an interaction radius of $7 \AA$ and $x=250 \mathrm{~cm} / \mathrm{s}$ one finds the rate constant for quenching to be $9.2 \times 109 \mathrm{M}^{-1} \mathrm{~s}^{-1}$. This value is similar to the bimolecular quenching constant of indole by either iodide or acrylamide, which from our data is about $7.1 \times 10^{9} \mathrm{M}^{-1} \mathrm{~s}^{-1}$. This indicates that the quenching reaction is faster than the diffusion-controlled encounter rate. It is possible that $\kappa$ is larger than $250 \mathrm{~cm} / \mathrm{s}$ but that the faster rate cannot be determined from the data because this rate does not limit the overall rate of quenching.

In summary, the frequency-domain data for both iodide and acrylamide quenching show clear deviations from the Smoluchowski moded. The radiation model appears to explain the iodide quenching data, but small deviations from this model have been detected for acrylamide quenching.

\section{Discussion}

The results and analyses indicate that the transient effects in collisional quenching are easily revealed by the frequency response of the fluorescence emission. Transient effects were first detected previously by time-correlated single photon counting. ${ }^{12}$ These workers examined the quenching of 1,2-benzanthracene by carbon tetrabromide in 1,2-propanediol. We suspect the transient effects were easier to detect in this case because of the 10-fold longer lifetime of 1,2-benzanthracene ( $40 \mathrm{~ns}$ ) and the 14- to 24-fold smaller diffusion coefficient expected for $\mathrm{CBr} 4$ in the more viscous solvent $\left(\sim 5 \times 10^{-7} \mathrm{~cm}^{2} / \mathrm{s}\right)$. It should also be noted that the shorter decay time of indole and especially that of the quenched indole samples would result in increased difficulty in the time domain due to the pulse width of the flash lamps. The difficulty is evident from the report by van Resandt, ${ }^{53}$ who studied the quenching of $\mathrm{N}$ acetyl-L-tryptophanamide by iodide using a 10-ps laser source, time-correlated single photon counting, and a microchannel plate photomultiplier. Even with $350 \mathrm{mM}$ iodide the intensity depays were barely distinguishable from a single exponential. The data were fit to the Smoluchowski model and did not provide any evidence for the inadequacy of this model. This comparison illustrates the sensitivity of the frequency-domain method to transient effects in quenching. In spite of the difficult nature of our samples, the transient effects were easily seen from the frequency responses, even to the extent of demonstrating deviations from the Smoluchowski model and possibly the radiation model.

The measured transient effects should be useful for comparison with theories for diffusion in liquids. To explain the data it may be necessary to use more complex models for diffusioncontrolled reactions. For instance, models have been described for the reaction between asymmetric molecules which react over only a limited region of their surfaces. ${ }^{37-39}$ The emphasis of these theories has been toward determining the rate constants at long times. ${ }^{40}$ For comparison with the present data it will be necessary to consider the transient effects for 
these less symmetric models, which has been accomplished to some extent. ${ }^{37-39}$

Alternatively, it may be preferable to shift away from such continuum models and to consider instead molecular dynamics simulations for a container of fluorophore, quencher, and solvent. Such simulations have been performed to study self-diffusion in simple liquids. 41-43 Simulations could be performed to predict the intensity decays, which could then be compared with the measured decays. Such simulations could include the effects of concerted motions, fractional reactivity, and rotational diffusion. Of course, lack of agreement would force a reevaluation of the assumptions used to accomplish the simulations.

And finally, quenching of fluorophores buried in proteins or embedded in membranes should contain transient terms which depend upon the diffusion rates of the quenchers within the macromolecules and the rates of transfer across the water-macromolecule interface. In fact, we recently observed that the intensity decays of several single tryptophan proteins become more heterogeneous in the presence of quenching by either oxygen or acrylamide. ${ }^{56}$ Such measurements may be compared with molecular dynamics simulations of quencher diffusion within proteins. ${ }^{57}$ Such data could also be compared with theories for diffusion within specific geometric boundaries. ${ }^{44-48}$

And finally it should be noted that the presence of transient effects due to quenching may invalidate some uses of quenching to recover subpopulations of fluorophores in macromolecules. For instance, lifetime measurements on progressively quenched proteins have been used to determine which populations of tryptophan residues are accessible to quenching. ${ }^{49,50}$ Such measurements have also been used to recover the individual emission spectra of the classes of tryptophan residues. ${ }^{50,51}$ In some cases it was noticed that the decays become more heterogeneous in the presence of quenching. For instance, Cheung et al. ${ }^{52}$ found that the decay of a labeled protein (myosin SI) was a single exponential in the absence of quencher. In the presence of either acrylamide or iodide the decay became more complex, as two decay times were needed to fit the data. The origin of the increased complexity was unknown, but the authors suggested the presence of two populations of fluorophores. Additionally, Weber and co-workers ${ }^{58,59}$ found that the intensity decays of a long-lived (20 ns) myoglobin derivative became multiexponential in the presence of oxygen quenching. This effect was attributed to two populations of protein molecules, with and without a resident oxygen molecule. It is not our intention to criticize these carefully performed studies. Rather, we wish to point out that the transient effects result in new and shorter lived components in the intensity decays. These components are revealed as shortlived components in the multiexponential analysis. Hence, the usual conclusion of greater quenching of the longer lived population may not always be correct. Instead, the transient effects may result in new components, which appear as shorter components from the multiexponential analysis.

\section{Acknowledgment.}

This work was supported by grants PCM-8210878 and DMB-08502835 from the National Science Foundation and GM-35154 from the National Institutes of Health to J.R.L. and by NIH grants AM 22125 and GM 28926 to M.L.J. I.G. expresses appreciation for financial support from Research Project CPBP 01.06.2.01 (Poland). 


\section{References}

(1). Lehner SS Biochemistry 1971, 10, 3254-3263. [PubMed: 5119250]

(2). Eftink MR; Ghiron CA J. Phys. Chem 1976, 80, 486-443.

(3). Eftink MR; Ghiron CA Anal. Biochem 1981, 114, 199-227. [PubMed: 7030122]

(4). Lakowicz JR; Weber G Biochemistry 1973, 12, 4161-4170. [PubMed: 4795686]

(5). Lakowicz JR; Weber G Biochemistry 1973, 12, 4171-4179. [PubMed: 4200894]

(6). Eftink MR; Ghiron CA Biochemistry 1976, 15, 672-680. [PubMed: 1252418]

(7). Eftink MR; Ghiron CA Biochemistry 1977, 16, 5546-5551. [PubMed: 921949]

(8). Lakowicz JR J. Biochem. Biophys. Methods 1980, 2, 90-119.

(9). Lakowicz JR; Hogen D Chem. Phys. Lipids 1980, 26, 1-40. [PubMed: 7357681]

(10). Frank JM; Vavilov SI Z. Phys 1931, 69, 100-110.

(11). Keizer JJ Am. Chem. Soc 1983, 105, 1494-1498.

(12). Ware WR; Nemzek TL Chem. Phys. Lett 1973, 23, 557-560.

(13). Nemzek TL; Ware WR J. Chem. Phys 1975, 62, 447-489.

(14). Ware WR; Novros JS J. Phys. Chem 1966, 70, 3246-3253.

(15). Gratton E; Linkeman M Biophys, J. 1983, 44, 315-324. [PubMed: 6661490]

(16). Lakowicz JR; Maliwal BP Biophys. Chem 1985, 21, 61-78. [PubMed: 3971026]

(17). Lakowicz JR; Laczko G; Gryczynski I Rev. Sci. Instrum 1986, 57, 2499-2506.

(18). Lakowicz JR; Laczko G; Gryczynski I Biochemistry 1987, 26, 82-90. [PubMed: 3828310]

(19). Gratton E; Linkeman M; Lakowicz JR; Maliwal BP; Cherek H; Laczko G Biophys. J 1984, 46, 463-477. [PubMed: 6498264]

(20). Lakowicz JR; Cherek H; Laczko G; Gratton E Biochim. Biophys. Acta 1984, 777, 183-193.

(21). Lakowicz JR; Cherek H Chem. Phys. Lett 1985,122, 380-384.

(22). Collins FC; Kimball GE J. Colloid Sci. 1949, 4, 425-437.

(23). Collins FC; J. Colloid Sci. 1950, 5, 499-505.This paper corrects an error in ref 22.

(24). Noyes RM Prog. React. Kinet 1961, 1, 129-160.

(25). Andre JC; Niclause M; Ware WR Chem. Phys 1978, 28, 371-377.

(26). Forsythe GE; Malcolm MA; Moler CB Computer Methods for Mathematical Computations; Prentice Hall: Englewood Cliffs, NJ, 1977.

(27). International Mathematics and Statistics Library.

(28). Bevington PR Data Reduction and Error Analysis for the Physical Sciences; McGraw-Hill: New York, 1969.

(29). johnson ML Biophys. J 1983, 44, 101-106. [PubMed: 6626675]

(30). Johnson ML; Frasier SG Methods Enzymol. 1985, 117, 301-342.

(31). Johnson ML; Lakowicz JR, manuscript in preparation.

(32). Lakowicz JR Principles of Fluorescence Spectroscopy, Plenum: New York, 1983.

(33). Eftink MR; Ghiron CA J. Phys. Chem 1976, 80, 486-493.

(34). Landolt-Bornstein. Transport Phenomenon-, Springer-Verlag: New York, 1967; II Band, 5 Tell, pp 642 and 650.

(35). International Critical Tables of Numerical Data, Physics, Chemistry and Technology., 19261930, Washburn EW, Editor-in-Chief; McGraw-Hill: New York, 1926-1930; p 70.

(36). Handbook of Chemistry and Physics., CRC: Boca Raton, FL, 1967; p F-45.

(37). Doi M Chem. Phys 1975, 11, 115-121.

(38). Doi M Chem. Phys 1975, 11, 107-113.

(39). Sole K; Stockmayer WH J. Chem. Phys 1971, 54, 2981-2988.

(40). Keizer JJ Phys. Chem 1982, 86, 5052-5067.

(41). Fehder PL J. Chem. Phys 1969, 50, 2617-2629.

(42). Fehder PL; Emeis CA; Futielle RP J. Chem. Phys 1971, 54, 4921-4934. 
(43). Emeis CA; Fehder PL J. Am. Chem. Soc 1970, 92, 2246-2252.

(44). Samson R; Deutch JM J. Chem. Phys 1978, 68, 285-290.

(45). Schmitz KS; Schurr JM J. Phys. Chem 1972, 76, 534-545.

(46). Sole K; Stockmayer WH int. J. Chem. Kinet 1973, 5, 733-752.

(47). Schurr JM; Schmitz KS J. Phys. Chem 1976, 80, 1934-1936.

(48). Keizer J Phys. Chem 1981, 85, 940-941.

(49). Eftink MR; Hagamon KA Biochemistry 1986, 25, 6631-6637. [PubMed: 3790548]

(50). Torgerson PM Biochemistry 1984, 23, 3002-3007. [PubMed: 6466627]

(51). Robbins DJ; Diebel MR; Barkley MD Biochemistry 1986, 24, 7250-7257.

(52). Cheung HC; Gonsoulin F; Garland FJ Biol. Chem 1983, 258, 5775-5786.

(53). van Resandt RW Chem. Phys. Lett 1983, 95, 205-208.

(54). Lakowicz JR; Cherek H; Balter AJ Biochem. Biophys. Methods 1981, 5, 131-146.

(55). Chang MC; Courtney S; Cross AJ; Gulotty RJ; Petrich JW; Fleming GR Anal. Instrum 1985, 433-464.

(56). Lakowicz JR; Johnson ML; Joshi N; Szmacinski H; Gryczynski JJ Biol. Chem, submitted for publication.

(57). Karplus M Methods Enzymol. 1986, 131, 283-307. [PubMed: 3773762]

(58). Jameson DM; Gratton E; Alpert B; Weber G Biophys. J 1984, 45, 795. [PubMed: 6722267]

(59). Gratton E; Jameson DM; Alpert B; Weber G Biophys. J 1984, 45, 789. [PubMed: 6722266] 

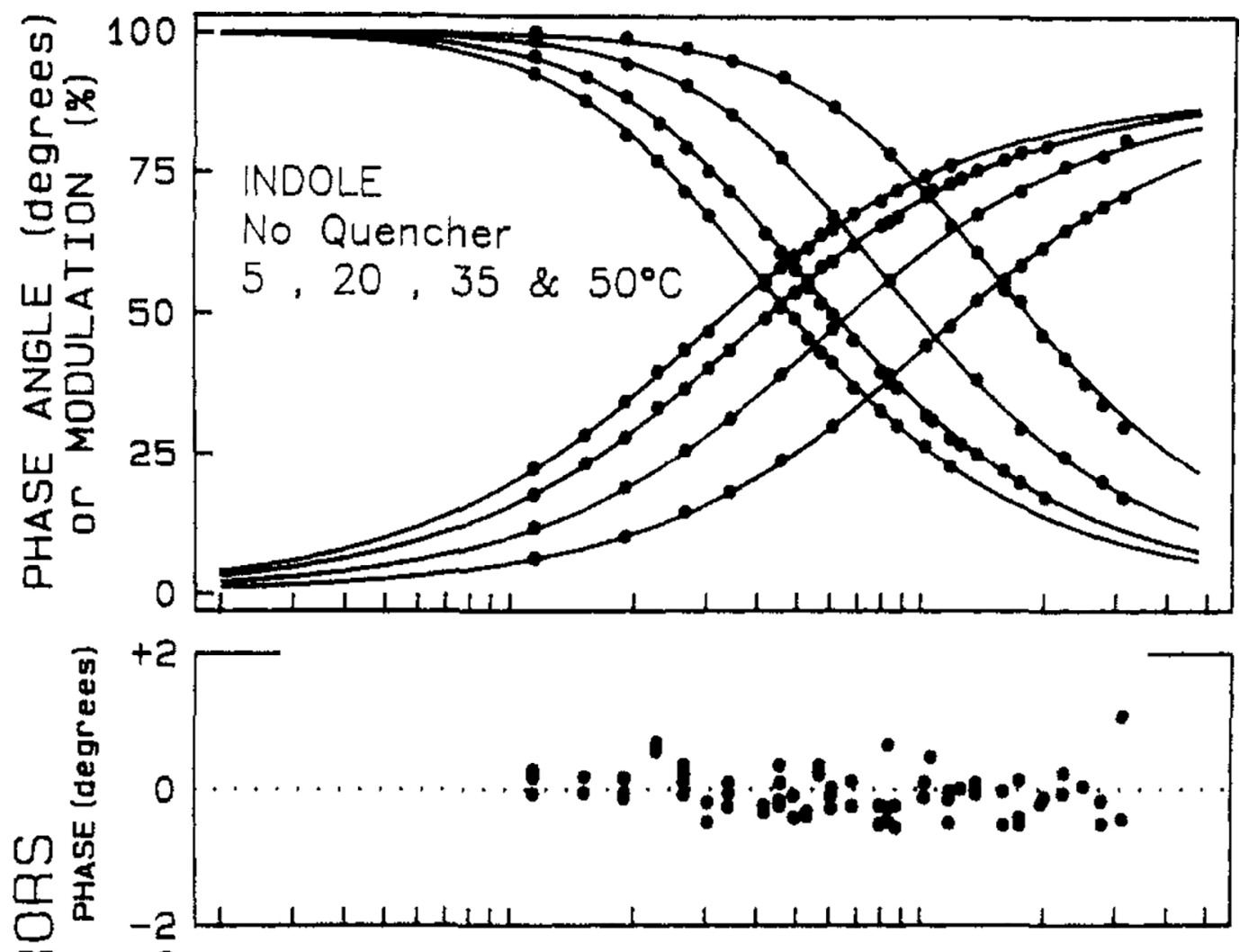

$\frac{\sigma}{\square}$
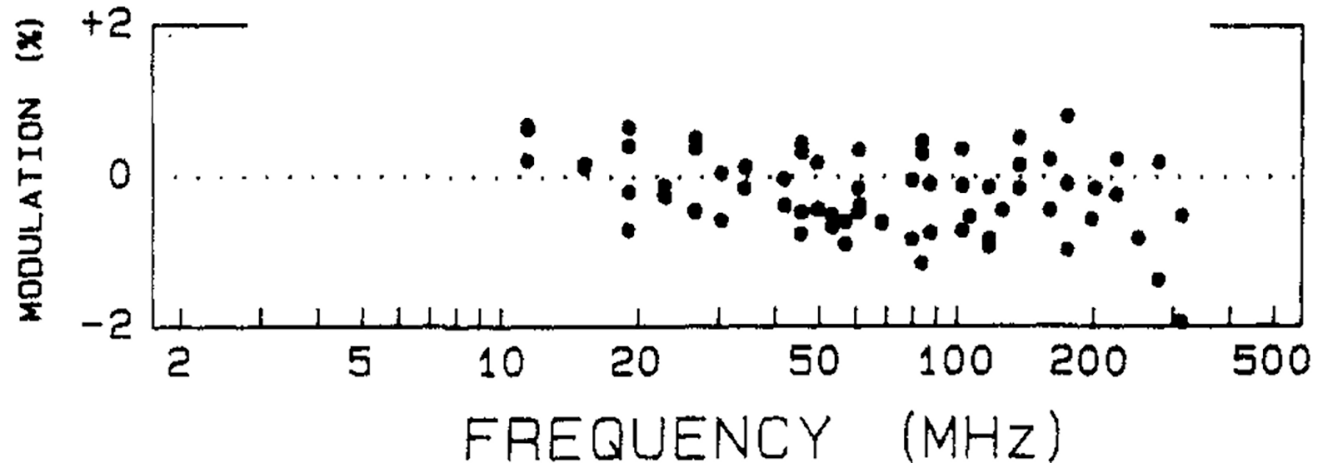

Figure 1.

Frequency-domain data for the intensity decay of indole in the absence of quencher. The upper panel shows the phase and modulation values $(-)$ for indole at $5,20,35$, and $50{ }^{\circ} \mathrm{C}$. The curves which increase with frequency are the phase angles, and the decreasing curves are the modulation values. As the temperature increases the lifetime of indole decreases, which shifts the frequency response to the right. The solid lines represent the best singleexponential decay fits to the data. The lower panels show the deviations between the data and the best fit. The decay parameters are summarized in Table I. 


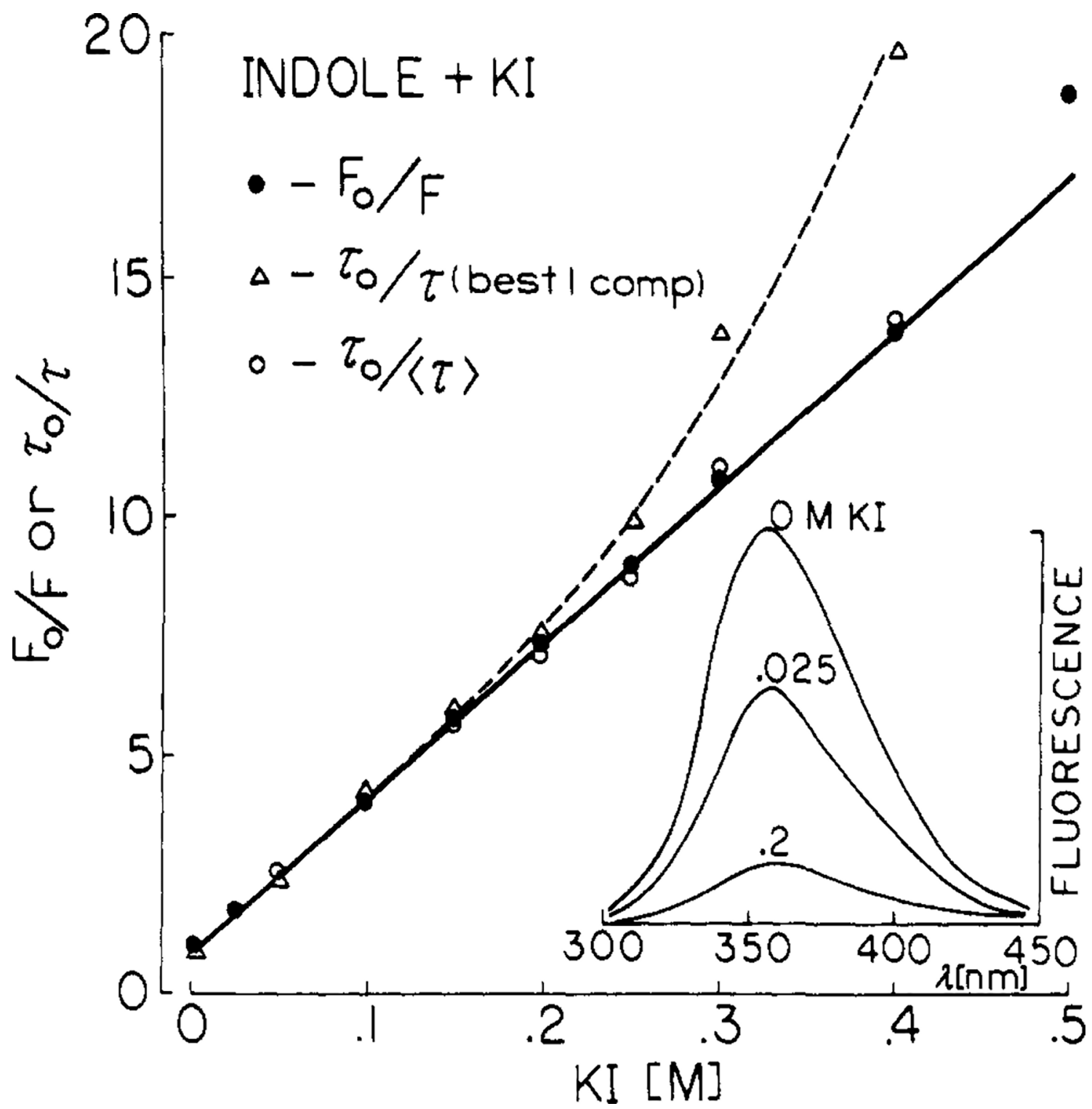

Figure 2.

Quenching of indole fluorescence by iodide, $20^{\circ} \mathrm{C}$. Stern-Volmer plots are shown for both the yields $(\bigcirc)$, the best-fit single decay times $(\Delta)$, and the mean decay time $(O)$ defined by $f_{1} \tau_{1}+f_{2} \tau_{2}$ (Table II). Inner filter effects are not significant for iodide with an excitation wavelength of $295 \mathrm{~nm}$. 


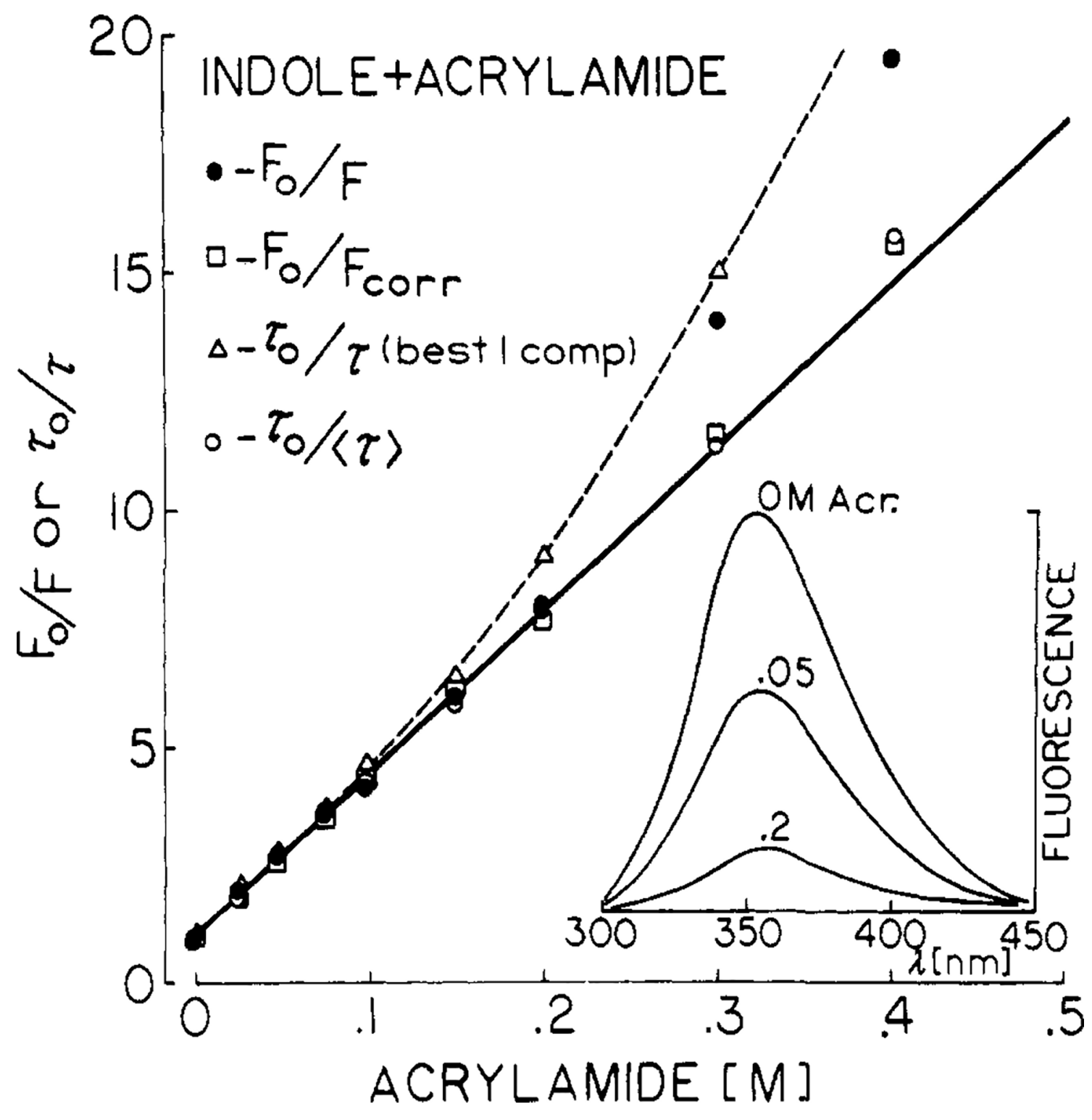

Figure 3.

Quenching of indole fluorescence by acrylamide, $20{ }^{\circ} \mathrm{C}$. Stern-Volmer plots are shown for the uncorrected yields $(\mathbf{O})$, the yields corrected for light absorption by acrylamide $(\square)$, the best single decay time $(\Delta)$, and the mean decay time $(O)$ defined by $f_{1} \tau_{1}+f_{2} \tau_{2}$ (Table III). 

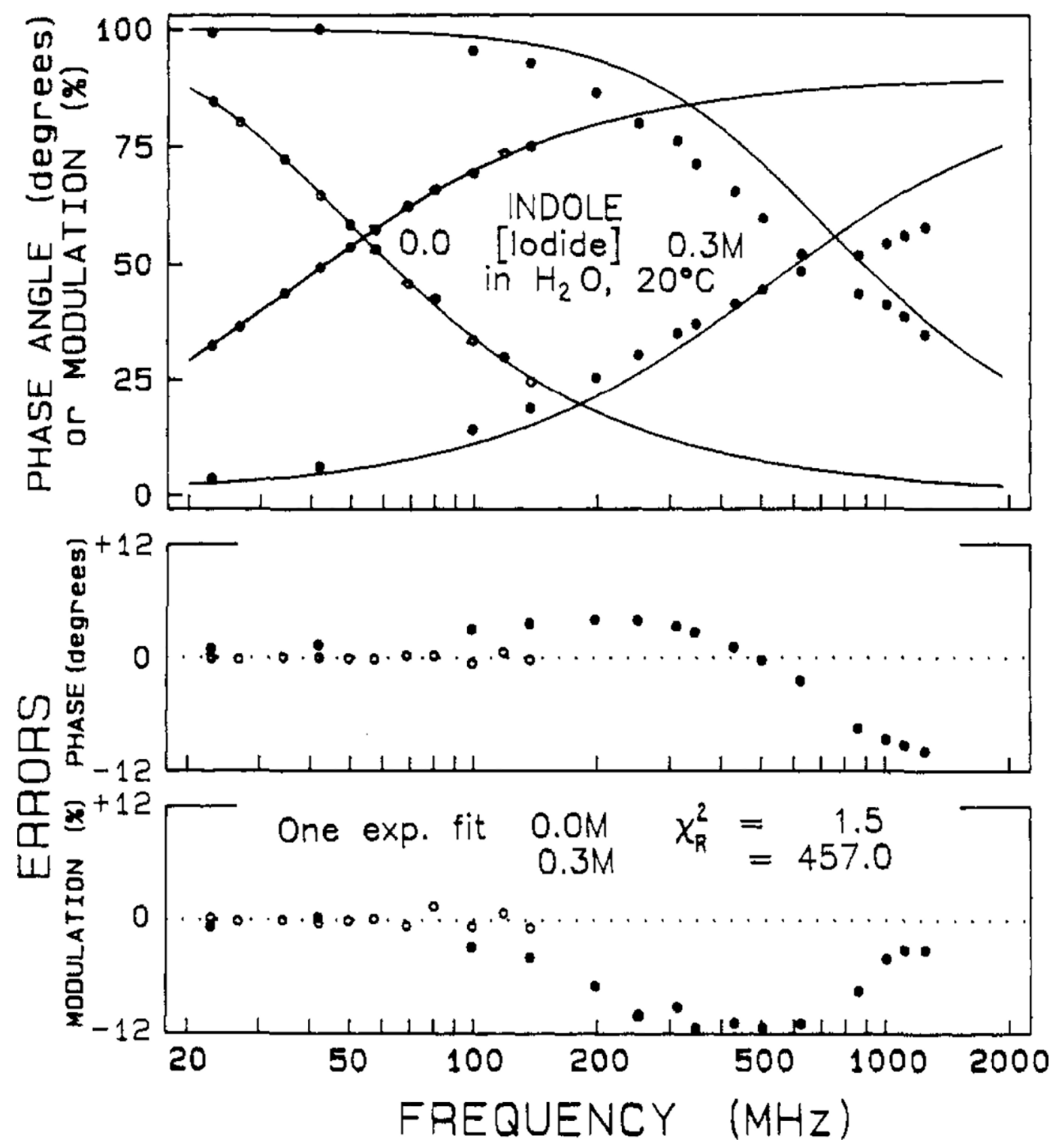

Figure 4.

Effect of iodide quenching on the intensity decay of indole. The solid lines represent the best single decay time fit through the data. The iodide concentrations are $0.0(\mathrm{O})$ and $0.3 \mathrm{M}(\bullet)$, $20^{\circ} \mathrm{C}$. The lower panels show the deviations from the best single decay time fit. 

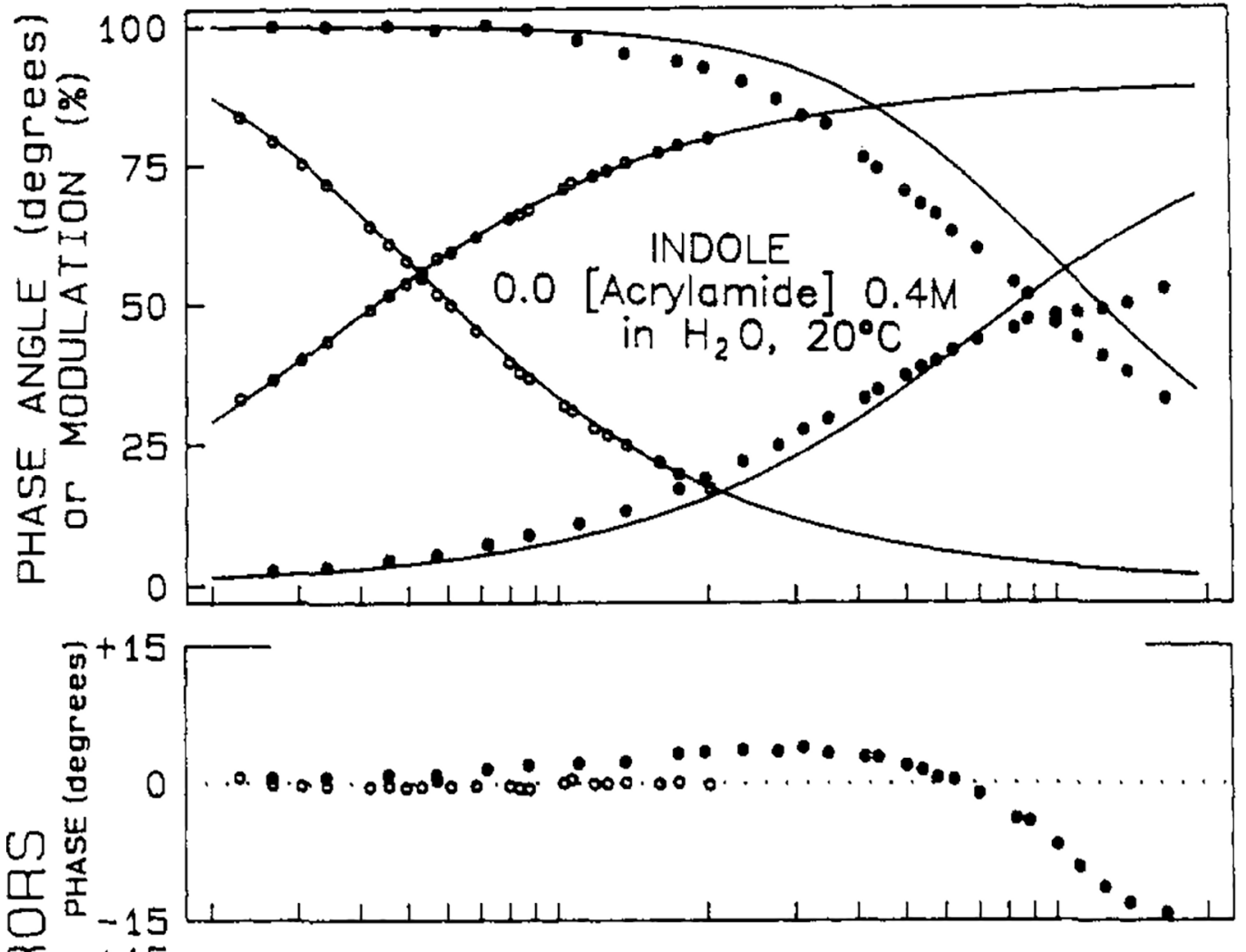

$$
\text { II }
$$

$\omega z$

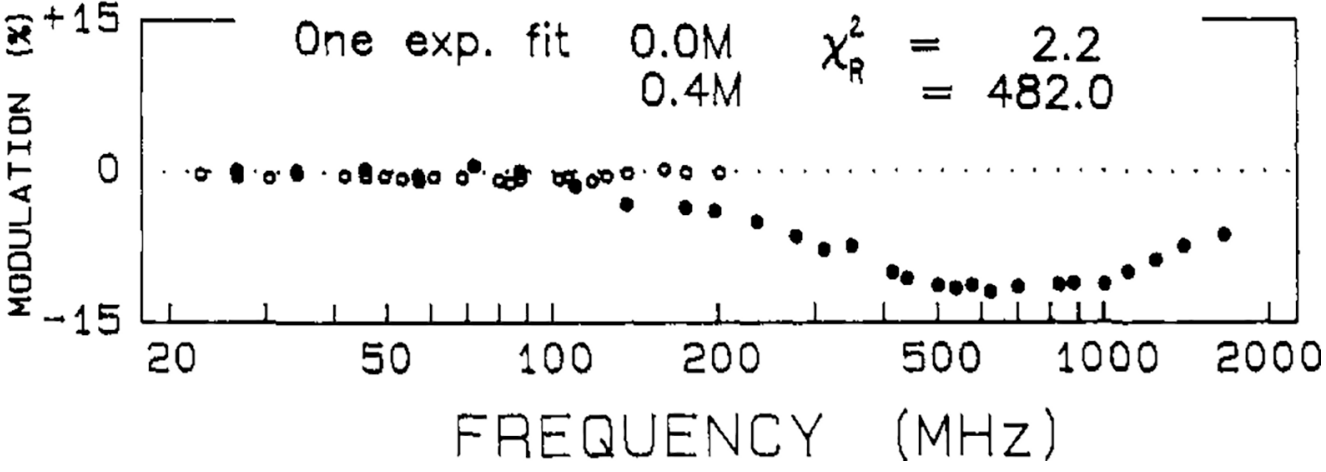

Figure 5.

Effect of acrylamide quenching on the intensity decay of indole. The solid lines represent the best single decay time fit to the data. The acrylamide concentrations are $0.0(O)$ and 0.4 $\mathrm{M}(\boldsymbol{O}), 20^{\circ} \mathrm{C}$. The lower panels show the deviations from the best single decay time fit. 

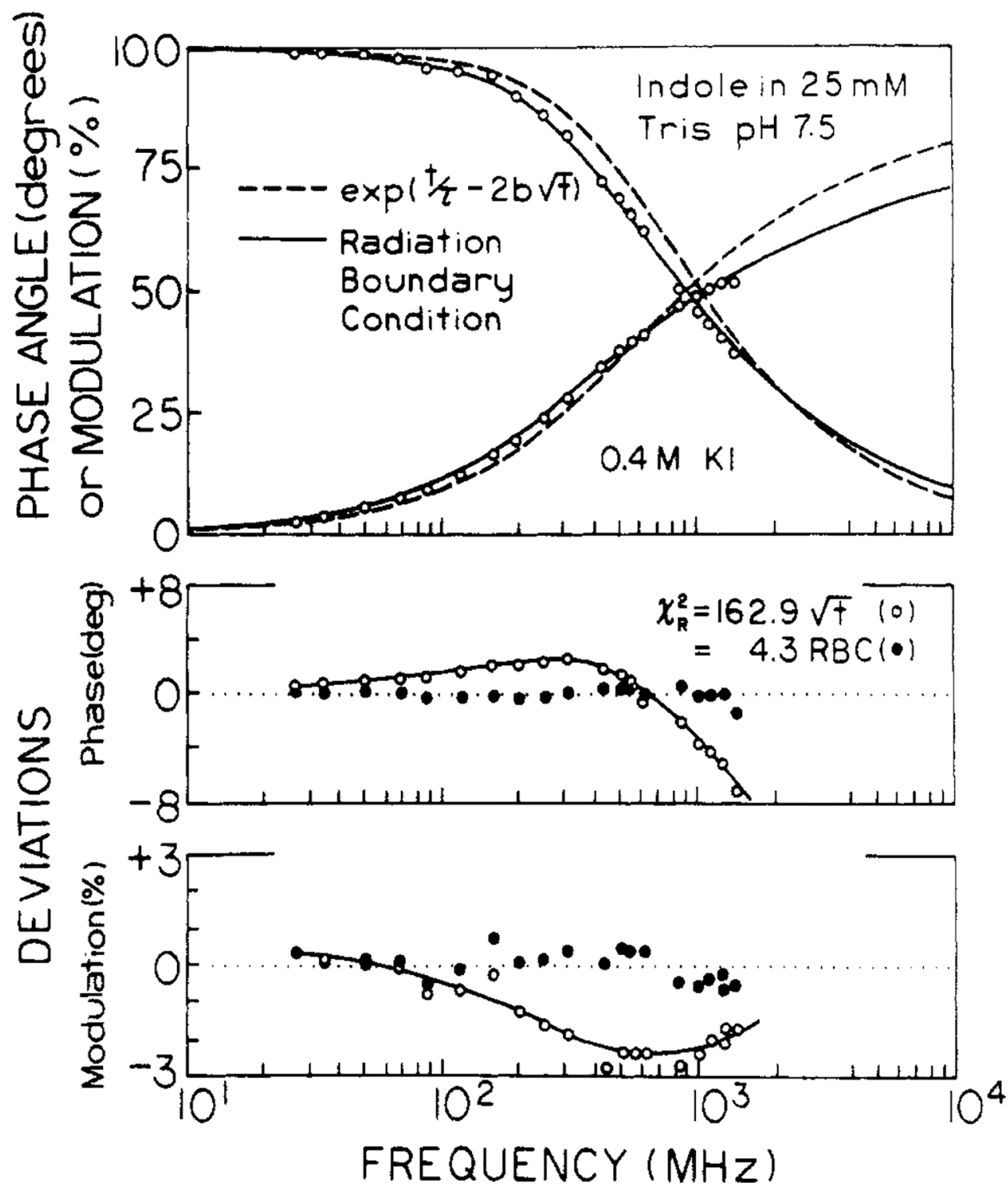

Figure 6.

Transient decay analysis of indole in the presence of $0.4 \mathrm{M}$ iodide. The data were analyzed by using the Smoluchowski model with $R$ fixed at $7 \AA$ (---, $\bigcirc)$ and with the radiation model $(-, \bigcirc)$ and with $\kappa=250 \mathrm{~cm} / \mathrm{s}$. The lower panels show the deviations $(\mathrm{O}, \mathrm{O})$. 

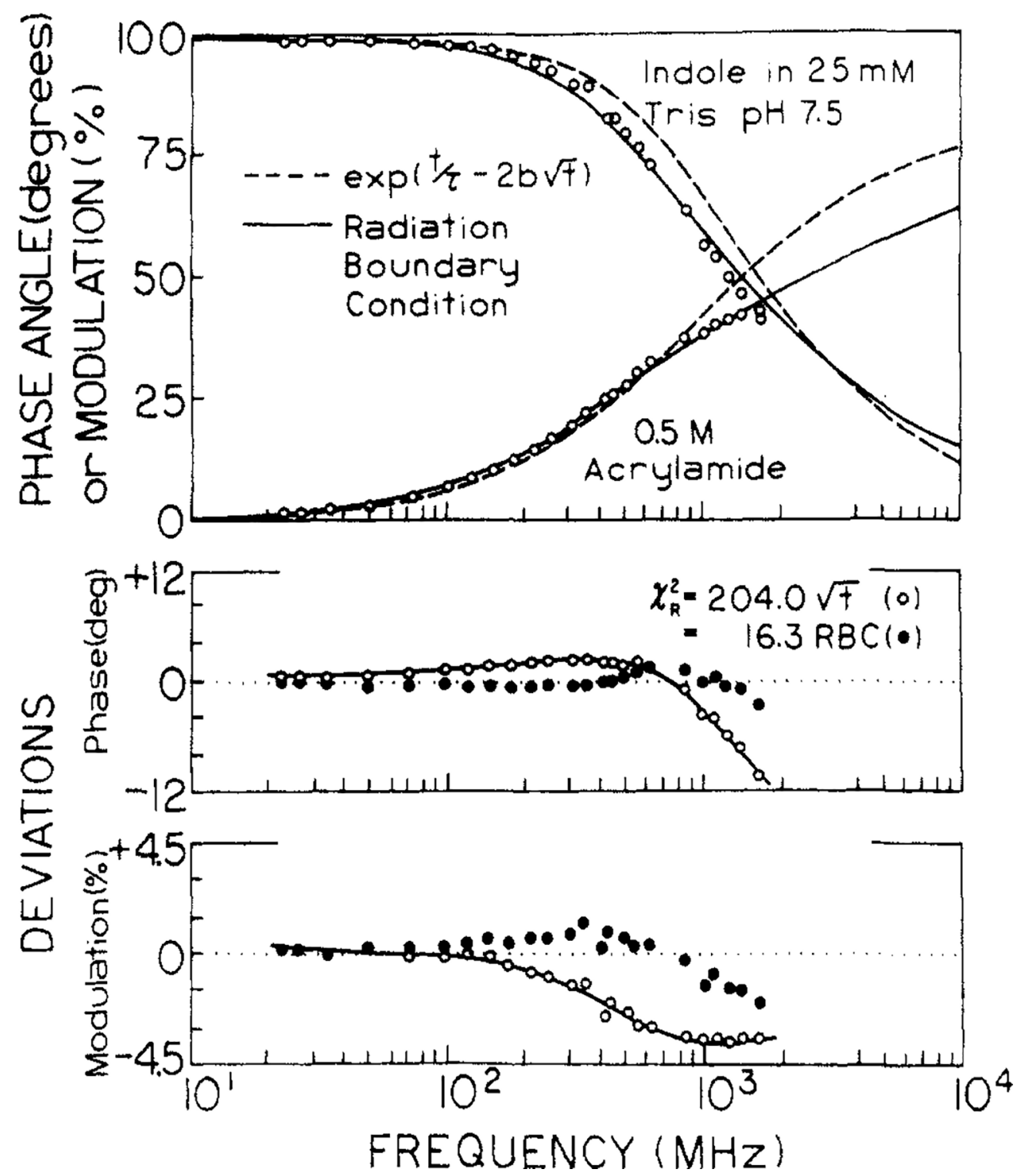

Figure 7.

Transient decay analysis of indole in the presence of $0.5 \mathrm{M}$ acrylamide. The data were analyzed by using the Smoluchowski model with $\mathrm{R}$ fixed at $7 \AA$ (---, O) and the radiation model with $\kappa=253 \mathrm{~cm} / \mathrm{s}$. The lower panels show the deviations $(\mathrm{O}, \mathbf{O})$. 
TABLE I:

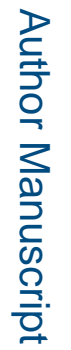

Intensity Decay Parameters for Indole in Water

\begin{tabular}{|c|c|c|c|c|}
\hline \multirow[b]{2}{*}{ temp, ${ }^{\circ} \mathrm{C}$} & \multicolumn{4}{|c|}{$\sum_{\alpha_{i} e^{-t / \tau_{i}}}(i=1$ or 2$)$} \\
\hline & $\tau_{i}, \mathbf{n s}$ & $a_{i}$ & $f_{i}$ & $\chi_{R}^{2}$ \\
\hline \multirow[t]{3}{*}{5} & 5.72 & 1.0 & 1.0 & 1.34 \\
\hline & $3.61(1.81-4.43)^{a}$ & 0.04 & 0.03 & \\
\hline & $5.82(5.75-5.85)$ & 0.96 & 0.97 & $1.05^{b}$ \\
\hline \multirow[t]{3}{*}{20} & 4.49 & 1.0 & 1.0 & 1.86 \\
\hline & $4.36(4.02-4.43)$ & 0.94 & 0.91 & \\
\hline & $6.55(5.23-7.41)$ & 0.06 & 0.09 & 1.61 \\
\hline \multirow[t]{3}{*}{35} & 2.85 & 1.0 & 1.0 & 2.65 \\
\hline & $2.81(2.73-2.91)$ & 0.96 & 0.95 & \\
\hline & $3.68(1.53-5.02)$ & 0.04 & 0.05 & 2.89 \\
\hline \multirow[t]{3}{*}{50} & 1.52 & 1.0 & 1.0 & 2.53 \\
\hline & $0.22(0-1.56)$ & 0.04 & 0.02 & \\
\hline & $1.53(1.51-1.56)$ & 0.96 & 0.98 & 2.22 \\
\hline
\end{tabular}

J Phys Chem. Author manuscript; available in PMC 2020 January 06. 


\section{TABLE II:}

Iodide Quenching of Indole in Water, Multiexponential Analysis

\begin{tabular}{|c|c|c|c|c|c|c|}
\hline \multirow[b]{3}{*}{ [iodide], mM } & \multirow{2}{*}{\multicolumn{2}{|c|}{$\exp (-t / \tau)$}} & \multicolumn{3}{|c|}{$-t / \tau_{1}$} & \multirow[t]{2}{*}{$t / \tau_{2}$} \\
\hline & & & $\alpha_{1} e$ & & $\alpha_{2} e$ & \\
\hline & $\tau, \mathbf{n s}$ & $\chi_{R}^{2}$ & $\tau_{i}, \mathrm{~ns}$ & $a_{i}$ & $f_{i}$ & $\chi_{R}^{2}$ \\
\hline \multirow[t]{2}{*}{0.0} & 4.45 & 1.5 & 4.36 & 0.94 & 0.91 & \\
\hline & & & 6.55 & 0.06 & 0.09 & 1.6 \\
\hline \multirow[t]{2}{*}{12.5} & 3.75 & 3.1 & 2.43 & 0.16 & 0.10 & \\
\hline & & & 3.98 & 0.84 & 0.90 & 0.96 \\
\hline \multirow[t]{2}{*}{25} & 2.53 & 12.5 & 0.20 & 0.15 & 0.01 & \\
\hline & & & 2.62 & 0.85 & 0.99 & 0.98 \\
\hline \multirow[t]{2}{*}{50} & 1.78 & 32.0 & 0.39 & 0.21 & 0.05 & \\
\hline & & & 1.95 & 0.79 & 0.95 & 1.03 \\
\hline \multirow[t]{2}{*}{75} & 1.32 & 35.4 & 0.30 & 0.23 & 0.06 & \\
\hline & & & 1.46 & 0.77 & 0.94 & 1.6 \\
\hline \multirow[t]{2}{*}{100} & 1.03 & 59.4 & 0.28 & 0.30 & 0.09 & \\
\hline & & & 1.20 & 0.70 & 0.91 & 1.4 \\
\hline \multirow[t]{2}{*}{150} & 0.77 & 108 & 0.21 & 0.36 & 0.11 & \\
\hline & & & 0.94 & 0.64 & 0.89 & 2.7 \\
\hline \multirow[t]{2}{*}{200} & 0.58 & 119 & 0.09 & 0.58 & 0.14 & \\
\hline & & & 0.76 & 0.42 & 0.86 & 2.6 \\
\hline \multirow[t]{2}{*}{250} & 0.43 & 260 & 0.09 & 0.58 & 0.16 & \\
\hline & & & 0.61 & 0.42 & 0.84 & 2.1 \\
\hline \multirow[t]{2}{*}{300} & 0.31 & 457 & 0.10 & 0.59 & 0.22 & \\
\hline & & & 0.50 & 0.41 & 0.78 & 2.1 \\
\hline \multirow[t]{2}{*}{400} & 0.23 & 474 & 0.06 & 0.62 & 0.21 & \\
\hline & & & 0.37 & 0.38 & 0.79 & 3.1 \\
\hline \multirow[t]{2}{*}{500} & 0.16 & 555 & 0.01 & 0.85 & 0.22 & \\
\hline & & & 0.26 & 0.15 & 0.78 & 3.8 \\
\hline
\end{tabular}

J Phys Chem. Author manuscript; available in PMC 2020 January 06. 


\section{TABLE III:}

Acrylamide Quenching of Indole in Water, Multiexponential Analysis

\begin{tabular}{|c|c|c|c|c|c|c|}
\hline \multirow[b]{3}{*}{ [acrylamide], mM } & \multirow{2}{*}{\multicolumn{2}{|c|}{$\exp (-t / \tau)$}} & \multicolumn{3}{|c|}{$-t / \tau_{1}$} & \multirow[t]{2}{*}{$t / \tau_{2}$} \\
\hline & & & $\alpha_{1} e^{2}$ & & $\alpha_{2} e$ & \\
\hline & $\tau$, ns & $\chi_{R}^{2}$ & $\tau_{i}, \mathbf{n s}$ & $a_{i}$ & $f_{i}$ & $\chi_{R}^{2}$ \\
\hline \multirow[t]{2}{*}{0} & 4.50 & 2.2 & 4.10 & 0.55 & 0.51 & \\
\hline & & & 4.98 & 0.45 & 0.49 & 2.1 \\
\hline \multirow[t]{2}{*}{25} & 2.42 & 7.3 & 1.14 & 0.13 & 0.06 & \\
\hline & & & 2.58 & 0.87 & 0.94 & 1.7 \\
\hline \multirow[t]{2}{*}{50} & 1.62 & 19.7 & 0.66 & 0.19 & 0.08 & \\
\hline & & & 1.79 & 0.83 & 0.92 & 1.6 \\
\hline \multirow[t]{2}{*}{75} & 1.19 & 42.2 & 0.42 & 0.22 & 0.08 & \\
\hline & & & 1.35 & 0.78 & 0.92 & 1.8 \\
\hline \multirow[t]{2}{*}{100} & 0.94 & 134 & 0.32 & 0.35 & 0.13 & \\
\hline & & & 1.18 & 0.65 & 0.87 & 2.0 \\
\hline \multirow[t]{2}{*}{150} & 0.68 & 165 & 0.15 & 0.31 & 0.08 & \\
\hline & & & 0.81 & 0.69 & 0.92 & 1.9 \\
\hline \multirow[t]{2}{*}{200} & 0.49 & 216 & 0.10 & 0.37 & 0.09 & \\
\hline & & & 0.61 & 0.63 & 0.91 & 2.5 \\
\hline \multirow[t]{2}{*}{300} & 0.31 & 424 & 0.08 & 0.51 & 0.16 & \\
\hline & & & 0.45 & 0.49 & 0.84 & 2.6 \\
\hline \multirow[t]{2}{*}{400} & 0.23 & 482 & 0.04 & 0.67 & 0.18 & \\
\hline & & & 0.34 & 0.33 & 0.82 & 2.8 \\
\hline \multirow[t]{2}{*}{500} & 0.15 & 444 & 0.01 & 0.84 & 0.22 & \\
\hline & & & 0.25 & 0.16 & 0.78 & 2.2 \\
\hline \multirow[t]{2}{*}{700} & 0.10 & 590 & 0.01 & 0.87 & 0.29 & \\
\hline & & & 0.21 & 0.13 & 0.71 & 2.21 \\
\hline
\end{tabular}

J Phys Chem. Author manuscript; available in PMC 2020 January 06. 


\section{로을}

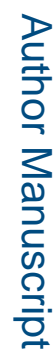

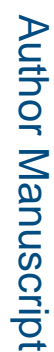

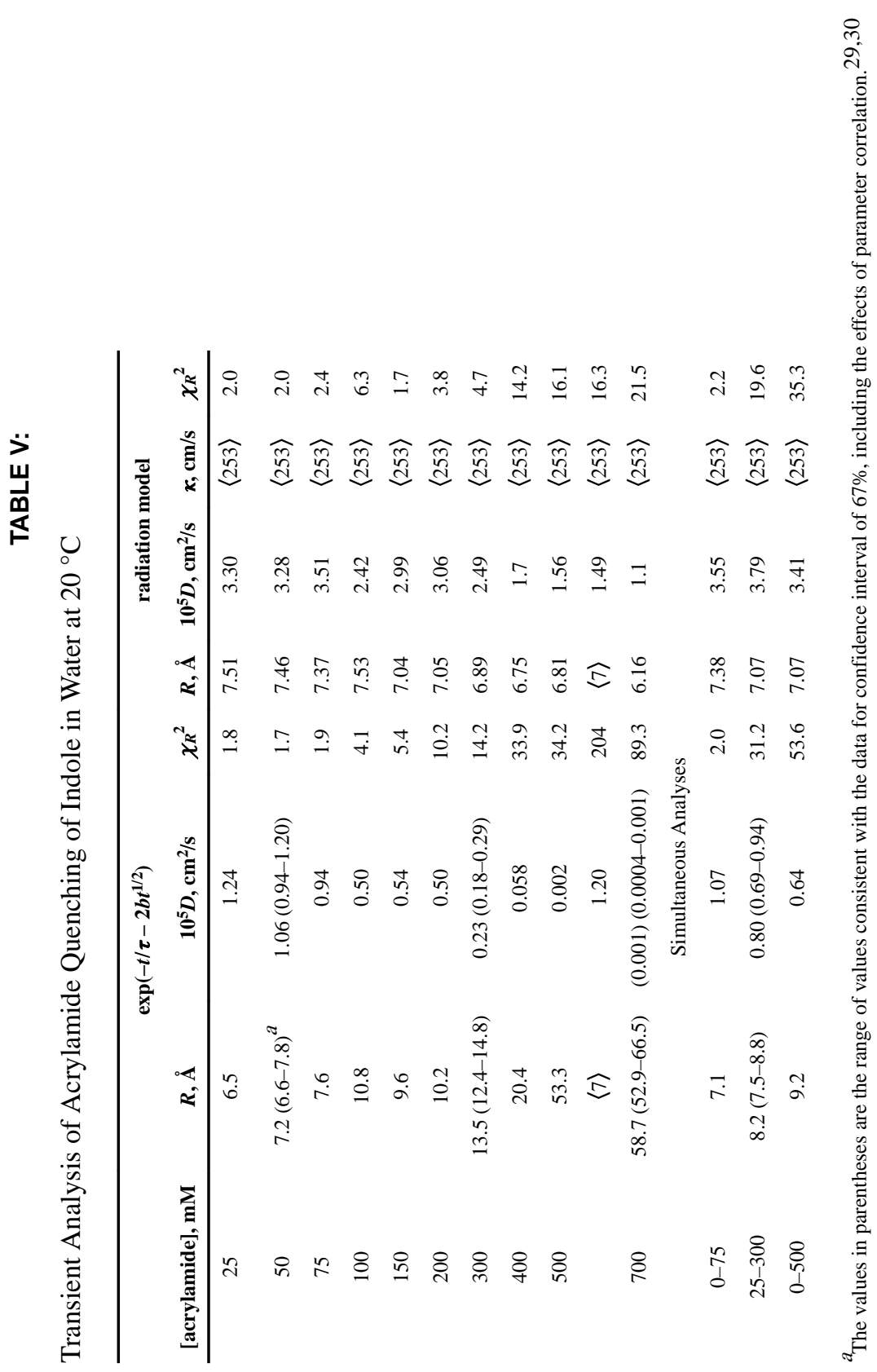

J Phys Chem. Author manuscript; available in PMC 2020 January 06 